\title{
ON THE SPECTRUM OF THE ONE-DIMENSIONAL SCHRÖDINGER HAMILTONIAN PERTURBED BY AN ATTRACTIVE GAUSSIAN POTENTIAL
}

\author{
Silvestro Fassari ${ }^{a, b, c, *}$, Manuel Gadella ${ }^{a}$, Luis Miguel Nieto $^{a}$, \\ FABIO RINALDi ${ }^{b, c}$ \\ a Departamento de Física Teórica, Atómica y Óptica, and IMUVA, Universidad de Valladolid, 47011 Valladolid, \\ Spain \\ ${ }^{b}$ CERFIM, PO Box 1132, Via F. Rusca 1, CH-6601 Locarno, Switzerland \\ ${ }^{c}$ Dipartimento di Fisica Nucleare, Subnucleare e delle Radiazioni, Universitá degli Studi Guglielmo Marconi, Via \\ Plinio 44, I-00193 Rome, Italy \\ * corresponding author: silvestro.fassari@uva.es
}

ABStRACT. We propose a new approach to the problem of finding the eigenvalues (energy levels) in the discrete spectrum of the one-dimensional Hamiltonian $H=-\partial_{x}^{2}-\lambda e^{-x^{2} / 2}$, by using essentially the well-known Birman-Schwinger technique. However, in place of the Birman-Schwinger integral operator we consider an isospectral operator in momentum space, taking advantage of the unique feature of this Gaussian potential, that is to say its invariance under Fourier transform. Given that such integral operators are trace class, it is possible to determine the energy levels in the discrete spectrum of the Hamiltonian as functions of $\lambda$ with great accuracy by solving a finite number of transcendental equations. We also address the important issue of the coupling constant thresholds of the Hamiltonian, that is to say the critical values of $\lambda$ for which we have the emergence of an additional bound state out of the absolutely continuous spectrum.

KEYWORDS: Schrödinger equation; Gaussian potential; Birman-Schwinger method; trace class operators; Fredholm determinant.

\section{INTRODUCTION}

Given its increasing relevance in the field of Nanophysics, it is particularly interesting to investigate the Schrödinger Hamiltonian with an attractive Gaussian potential $V=-\lambda e^{-x^{2} / 2}$, since the latter has the typical properties of short-range potentials, which implies the existence of bound states with negative energies below the absolutely continuous spectrum given by the semibounded interval $[0,+\infty)$, but also those of the harmonic oscillator near the bottom of the well. Hence, the original Schrödinger eigenvalue problem we are going to consider is

$$
\left(-\frac{\mathrm{d}^{2}}{\mathrm{~d} x^{2}}-\lambda e^{-x^{2} / 2}\right) \psi=-\epsilon^{2} \psi, \quad \epsilon>0
$$

Although there have been quite a few papers on this model, the most rigorous of which being [1] by F. M. Fernández (see also [2, 3]), it seems that the implications of a remarkable feature of this potential, namely its invariance with respect to the Fourier transform, have been missed. Our method, whilst being essentially based on the (by now) classical Birman-Schwinger method (see, e.g., 4, 5]), considers instead of the integral operator associated to 11

$$
\lambda e^{-x^{2} / 4}\left(-\frac{\mathrm{d}^{2}}{\mathrm{~d} x^{2}}+\epsilon^{2}\right)^{-1} e^{-x^{2} / 4}
$$

another integral operator which is isospectral to the Birman-Schwinger one given in (2] (see [6] [8]):

$$
\lambda B_{\epsilon}=\lambda\left(-\frac{\mathrm{d}^{2}}{\mathrm{~d} x^{2}}+\epsilon^{2}\right)^{-1 / 2} e^{-x^{2} / 2}\left(-\frac{\mathrm{d}^{2}}{\mathrm{~d} x^{2}}+\epsilon^{2}\right)^{-1 / 2}
$$

Given that the function $e^{-x^{2} / 2}$ is invariant under the Fourier transform, the unitary equivalent of the above integral operator, still denoted by $B_{\epsilon}$, is

$$
B_{\epsilon}=\frac{1}{\sqrt{2 \pi}}\left(p^{2}+\epsilon^{2}\right)^{-1 / 2} e^{-p^{2} / 2} *\left(p^{2}+\epsilon^{2}\right)^{-1 / 2},
$$


where the star denotes the convolution. Therefore, the Schrödinger equation (1) can be reformulated in terms of the following integral equation:

$$
\chi(p)=\frac{1}{\sqrt{2 \pi}}\left(p^{2}+\epsilon^{2}\right)^{-1 / 2} \int_{-\infty}^{+\infty} e^{-(p-q)^{2} / 2}\left(q^{2}+\epsilon^{2}\right)^{-1 / 2} \chi(q) \mathrm{d} q,
$$

with $\widehat{\psi}(p)=\left(p^{2}+\epsilon^{2}\right)^{-1 / 2} \chi(p)$, in the sense that if $\epsilon$ is a value for which the above integral operator has an eigenvalue equal to one, then $-\epsilon^{2}$ is an eigenvalue of the original Schrödinger equation. As a consequence, understanding in depth the properties of the integral operator is crucial in order to get a detailed description of the discrete spectrum of the original Hamiltonian.

\section{The integral operator $B_{\epsilon}$}

We wish to open this section by stating and proving the key property of the integral operator $B_{\epsilon}$.

Theorem 2.1. The integral operator $B_{\epsilon}$ is trace class.

Proof. As a consequence of a well-known theorem (see [9]), given the positivity of the integral kernel and its smoothness, the trace is simply given by the integral of the kernel evaluated along the diagonal $p=q$, that is to say:

$$
\frac{1}{\sqrt{2 \pi}} \int_{-\infty}^{+\infty} \frac{\mathrm{d} p}{p^{2}+\epsilon^{2}}=\sqrt{\frac{\pi}{2}} \frac{1}{\epsilon}
$$

The fact that the trace diverges as $\epsilon \rightarrow 0^{+}$, guarantees that there is always at least one bound state even for very small values of the coupling constant (shallow wells). We take this opportunity to remind the reader that the latter property is typical of one-dimensional quantum Hamiltonians, as shown in [5, 10]. We also notice that, by expanding the square in the exponent of the Gaussian in $(5)$, the integral kernel $B_{\epsilon}(p, q)$ of the operator can be recast as:

$$
B_{\epsilon}(p, q)=\frac{1}{\sqrt{2 \pi}} \frac{e^{-p^{2} / 2}}{\left(p^{2}+\epsilon^{2}\right)^{1 / 2}} e^{p q} \frac{e^{-q^{2} / 2}}{\left(q^{2}+\epsilon^{2}\right)^{1 / 2}},
$$

As an immediate consequence of (7), the operator $B_{\epsilon}$ can be written as a direct sum of two operators, one acting onto the symmetric subspace $(s)$ the other onto the antisymmetric one $(a)$, whose kernels are:

$$
\begin{aligned}
& B_{\epsilon}^{s}(p, q)=\frac{1}{\sqrt{2 \pi}} \frac{e^{-p^{2} / 2}}{\left(p^{2}+\epsilon^{2}\right)^{1 / 2}} \cosh (p q) \frac{e^{-q^{2} / 2}}{\left(q^{2}+\epsilon^{2}\right)^{1 / 2}}, \\
& B_{\epsilon}^{a}(p, q)=\frac{1}{\sqrt{2 \pi}} \frac{e^{-p^{2} / 2}}{\left(p^{2}+\epsilon^{2}\right)^{1 / 2}} \sinh (p q) \frac{e^{-q^{2} / 2}}{\left(q^{2}+\epsilon^{2}\right)^{1 / 2}} .
\end{aligned}
$$

By using the Taylor expansion for each hyperbolic function, the two integral operators can be written as infinite sums of rank one operators, namely:

$$
\begin{gathered}
B_{\epsilon}^{s}=\frac{1}{\sqrt{2 \pi}} \sum_{n=0}^{\infty} \frac{1}{(2 n) !}\left|\frac{p^{2 n} e^{-p^{2} / 2}}{\left(p^{2}+\epsilon^{2}\right)^{1 / 2}}\right\rangle\left\langle\frac{p^{2 n} e^{-p^{2} / 2}}{\left(p^{2}+\epsilon^{2}\right)^{1 / 2}}\right|, \\
B_{\epsilon}^{a}=\frac{1}{\sqrt{2 \pi}} \sum_{n=0}^{\infty} \frac{1}{(2 n+1) !}\left|\frac{p^{2 n+1} e^{-p^{2} / 2}}{\left(p^{2}+\epsilon^{2}\right)^{1 / 2}}\right\rangle\left\langle\frac{p^{2 n+1} e^{-p^{2} / 2}}{\left(p^{2}+\epsilon^{2}\right)^{1 / 2}}\right| .
\end{gathered}
$$

Obviously, as a consequence of (6), both operators are trace class, which implies that both series are convergent in the trace class norm. Furthermore, although either operator has not yet been diagonalised at this stage (because the rank one operators are not mutually orthogonal), their diagonalisation can be achieved starting from the first rank one operator in each expansion $(n=0)$, that is to say:

$$
\begin{aligned}
& B_{\epsilon}^{s, 0}=\frac{1}{\sqrt{2 \pi}}\left|\frac{e^{-p^{2} / 2}}{\left(p^{2}+\epsilon^{2}\right)^{1 / 2}}\right\rangle\left\langle\frac{e^{-p^{2} / 2}}{\left(p^{2}+\epsilon^{2}\right)^{1 / 2}}\right|, \\
& B_{\epsilon}^{a, 0}=\frac{1}{\sqrt{2 \pi}}\left|\frac{p e^{-p^{2} / 2}}{\left(p^{2}+\epsilon^{2}\right)^{1 / 2}}\right\rangle\left\langle\frac{p e^{-p^{2} / 2}}{\left(p^{2}+\epsilon^{2}\right)^{1 / 2}}\right| .
\end{aligned}
$$

Their norms can be easily computed as the required improper integrals are well known:

$$
\begin{gathered}
\left\|B_{\epsilon}^{s, 0}\right\|=\frac{1}{\sqrt{2 \pi}} \int_{-\infty}^{+\infty} \frac{e^{-p^{2}}}{p^{2}+\epsilon^{2}} \mathrm{~d} p=\sqrt{\frac{\pi}{2 \epsilon^{2}}} e^{\epsilon^{2}} \operatorname{erfc}(\epsilon) \\
\left\|B_{\epsilon}^{a, 0}\right\|=\frac{1}{\sqrt{2 \pi}} \int_{-\infty}^{+\infty} \frac{p^{2} e^{-p^{2}}}{p^{2}+\epsilon^{2}} \mathrm{~d} p=\frac{1}{\sqrt{2}}\left(1-\sqrt{\pi} \epsilon e^{\epsilon^{2}} \operatorname{erfc}(\epsilon)\right) .
\end{gathered}
$$


As can be understood from (14), the divergence of the trace of the entire operator as $\epsilon \rightarrow 0^{+}$is only due to the the divergence of $B_{\epsilon}^{s, 0}$. In fact, the trace class norm of the positive operator $B_{\epsilon}^{s, 1}=B_{\epsilon}^{s}-B_{\epsilon}^{s, 0}$ can be calculated as follows:

$$
\left\|B_{\epsilon}^{s, 1}\right\|_{1}=\frac{1}{\sqrt{2 \pi}} \int_{-\infty}^{+\infty} \frac{e^{-p^{2}}\left(\cosh p^{2}-1\right)}{p^{2}+\epsilon^{2}} \mathrm{~d} p .
$$

By writing the hyperbolic cosine as a combination of two exponentials, the right hand side of 16 is easily seen to be equal to

$$
\left\|B_{\epsilon}^{s, 1}\right\|_{1}=\sqrt{\frac{\pi}{2^{3} \epsilon^{2}}}\left(1+e^{2 \epsilon^{2}} \operatorname{erfc}\left(2^{1 / 2} \epsilon\right)-2 e^{\epsilon^{2}} \operatorname{erfc}(\epsilon)\right)
$$

which, given its removable singularity at the origin, is almost immediately seen to converge to $\sqrt{2}-1$ as $\epsilon \rightarrow 0^{+}$.

Before determining the equation that will enable us to compute the ground state energy as a function of the coupling parameter $\lambda$, let us consider also the antisymmetric component $B_{\epsilon}^{a}$. As a result of the explicit expression of the integral kernel of $B_{\epsilon}^{a}$, it is not difficult to show that the latter operator converges weakly to $B_{0}^{a}$, where

$$
B_{0}^{a}(p, q)=\frac{1}{\sqrt{2 \pi}} \frac{e^{-p^{2} / 2}}{|p|} \sinh (p q) \frac{e^{-q^{2} / 2}}{|q|} .
$$

The latter is a positive trace class operator with trace equal to

$$
\left\|B_{0}^{a}\right\|_{1}=\frac{1}{\sqrt{2 \pi}} \int_{-\infty}^{+\infty} \frac{e^{-p^{2}}}{p^{2}} \sinh p^{2} \mathrm{~d} p=1,
$$

as follows easily by writing the hyperbolic sine as a combination of two exponentials and using integration by parts. It is worth pointing out that the latter quantity is nothing else but the limit, as $\epsilon \rightarrow 0^{+}$of the trace class norm of $B_{\epsilon}^{a}$, since

$$
\left\|B_{\epsilon}^{a}\right\|_{1}=\frac{1}{\sqrt{2 \pi}} \int_{-\infty}^{+\infty} \frac{e^{-p^{2}} \sinh p^{2}}{p^{2}+\epsilon^{2}} \mathrm{~d} p=\sqrt{\frac{\pi}{2^{3} \epsilon^{2}}}\left(1-e^{2 \epsilon^{2}} \operatorname{erfc}(\sqrt{2} \epsilon)\right),
$$

which ensures the convergence in the norm topology of trace class operators, as a consequence of a well-known theorem on operators belonging not only to the trace class $\mathcal{T}_{1}$ but to any ideal $\mathcal{T}_{p}$ (see [11]). This result can be summarised in the following statement.

Theorem 2.2. The positive trace class operator $B_{\epsilon}^{a}$ converges, as $\epsilon \rightarrow 0^{+}$, to $B_{0}^{a}$, the positive trace class operator defined by its kernel in (18), in the norm topology of trace class operators.

It is crucial to point out that, whilst $B_{\epsilon}^{s, 0}$, the first rank one summand in the expansion of the symmetric component of our integral operator, diverges as $\epsilon \rightarrow 0^{+}, B_{\epsilon}^{a, 0}$ obviously converges to the following rank one operator:

$$
B_{0}^{a 0}=\frac{1}{\sqrt{2 \pi}}\left|\frac{p e^{-p^{2} / 2}}{|p|}\right\rangle\left\langle\frac{p e^{-p^{2} / 2}}{|p|}\left|=\frac{1}{\sqrt{2 \pi}}\right| \operatorname{sgn}(p) e^{-p^{2} / 2}\right\rangle\left\langle\operatorname{sgn}(p) e^{-p^{2} / 2}\right| .
$$

Although the antisymmetric function $\operatorname{sgn}(p) e^{-p^{2} / 2}$ has a jump discontinuity at the origin, its square coincides with the one of the unnormalised ground state eigenfunction of the harmonic oscillator in momentum space.

\section{The tWo LOWEST EigenVAlues OF $-\partial_{x}^{2}-\lambda e^{-x^{2} / 2}$}

\subsection{The ground State}

As pointed out earlier, the divergent behaviour of the first term $(n=0)$ of the expansion of the symmetric part (12) implies that, no matter how shallow the Gaussian well may be (small values of the coupling constant $\lambda$ ), there will always exist at least one bound state, the ground state, whose energy is $-\epsilon_{0}(\lambda)^{2}$ with $\epsilon_{0}(\lambda)$ given by the solution of a transcendental equation that can be derived from the application of well-known facts regarding the Fredholm determinant of a trace class operator (see, e.g., [12]), namely:

$$
\operatorname{det}\left(1-\lambda B_{\epsilon}^{s}\right)=0
$$

By isolating the first divergent rank one operator in the expansion of $\lambda B_{\epsilon}^{s}$ and taking advantage of the boundedness of $\lambda B_{\epsilon}^{s, 1}$, the left hand side of 22 can be rewritten as

$$
\operatorname{det}\left(1-\lambda B_{\epsilon}^{s, 0}\left(1-\lambda B_{\epsilon}^{s, 1}\right)^{-1}\right) \operatorname{det}\left(1-\lambda B_{\epsilon}^{s, 1}\right) .
$$




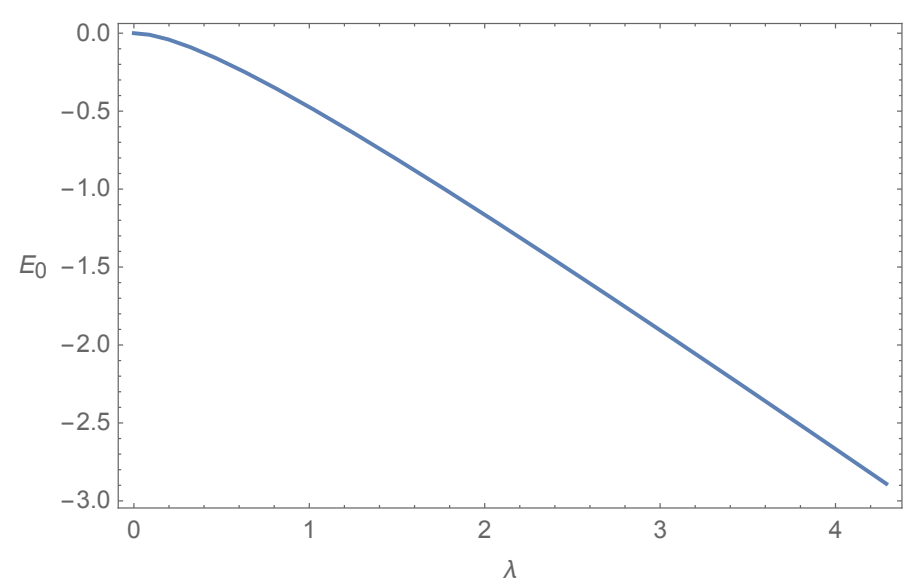

Figure 1. The ground state energy $E_{0}(\lambda)=-\epsilon_{0}(\lambda)^{2}$, as a function of the coupling parameter $\lambda$.

As the first factor is the only one involving the divergent rank one operator $B_{\epsilon}^{s, 0}$, the equation leading to the determination of the ground state energy reduces to

$$
\operatorname{det}\left(1-\lambda B_{\epsilon}^{s, 0}\left(1-\lambda B_{\epsilon}^{s, 1}\right)^{-1}\right)=1-\operatorname{tr}\left(\lambda B_{\epsilon}^{s, 0}\left(1-\lambda B_{\epsilon}^{s, 1}\right)^{-1}\right)=0,
$$

given that the second term inside the deteminant is a rank one operator.

By taking only the terms up to $\lambda$ in the expansion of the inverse inside the trace in (24), we get the following quadratic equation in $\lambda$ :

$$
\frac{g_{0}(\epsilon)}{2 \pi} \lambda^{2}+\frac{f_{0}(\epsilon)}{\sqrt{2 \pi}} \lambda-1=0
$$

with

$$
\begin{gathered}
f_{0}(\epsilon)=\int_{-\infty}^{+\infty} \frac{e^{-p^{2}}}{p^{2}+\epsilon^{2}} \mathrm{~d} p=\frac{\pi}{\epsilon} e^{\epsilon^{2}} \operatorname{erfc}(\epsilon) \\
g_{0}(\epsilon)=\int_{-\infty}^{+\infty} \mathrm{d} p \int_{-\infty}^{+\infty} \frac{e^{-p^{2}}}{p^{2}+\epsilon^{2}}(\cosh (p q)-1) \frac{e^{-q^{2}}}{q^{2}+\epsilon^{2}} \mathrm{~d} q
\end{gathered}
$$

By using the standard Taylor expansion of the hyperbolic cosine, the latter double integral can be recast as the following convergent series whose coefficients are expressed in terms of the Gamma and the incomplete Gamma functions:

$$
g_{0}(\epsilon)=\sum_{n=1}^{\infty} \frac{1}{(2 n) !}\left(\int_{-\infty}^{+\infty} \frac{p^{2 n} e^{-p^{2}}}{p^{2}+\epsilon^{2}} \mathrm{~d} p\right)^{2}=e^{2 \epsilon^{2}} \sum_{n=1}^{\infty} \epsilon^{2(2 n-1)} \frac{\left(\Gamma(n+1 / 2) \Gamma\left(-n+1 / 2, \epsilon^{2}\right)\right)^{2}}{\Gamma(2 n+1)} .
$$

Hence, the positive solution of $(25)$ is given by the function from $[0,+\infty)$ to $[0,+\infty)$ :

$$
\lambda(\epsilon)=\frac{2 \sqrt{2 \pi}}{\left(f_{0}^{2}(\epsilon)+4 g_{0}(\epsilon)\right)^{1 / 2}+f_{0}(\epsilon)},
$$

which can be inverted to get the required $\epsilon_{0}(\lambda)$, leading to the ground state energy $E_{0}(\lambda)=-\epsilon_{0}(\lambda)^{2}$, the plot of which is shown in Figure 1 .

\subsection{The FIRST EXCITED STATE}

Let us now consider the antisymmetric component given by $(9)$ or $(11)$, so that we have to study the equation $\operatorname{det}\left(1-\lambda B_{\epsilon}^{a}\right)=0$. As a result of the explicit expression of the integral kernel of $B_{\epsilon}^{a}$, it is not difficult to show that $\lambda B_{\epsilon}^{a}$ converges weakly to $\lambda B_{0}^{a}$, where the trace class operator $B_{0}^{a}$ is given by (18).

As a consequence of Theorem 2.2 and in analogy to what has been done for the determination of the ground state energy, $E_{1}(\lambda)=-\epsilon_{1}(\lambda)^{2}$, the energy of the first antisymmetric bound state, can be determined by means of the following equation:

$$
\operatorname{det}\left(1-\lambda B_{\epsilon}^{a, 0}\left(I-\lambda B_{\epsilon}^{a, 1}\right)^{-1}\right)=0
$$

with $B_{\epsilon}^{a, 1}=B_{\epsilon}^{a}-B_{\epsilon}^{a, 0}$, which is obviously trace class. Taking account of the fact that $B_{\epsilon}^{a, 0}$ is a rank one operator, the latter equation becomes

$$
1-\lambda \operatorname{tr}\left(B_{\epsilon}^{a, 0}\left(I-\lambda B_{\epsilon}^{a, 1}\right)^{-1}\right)=0
$$




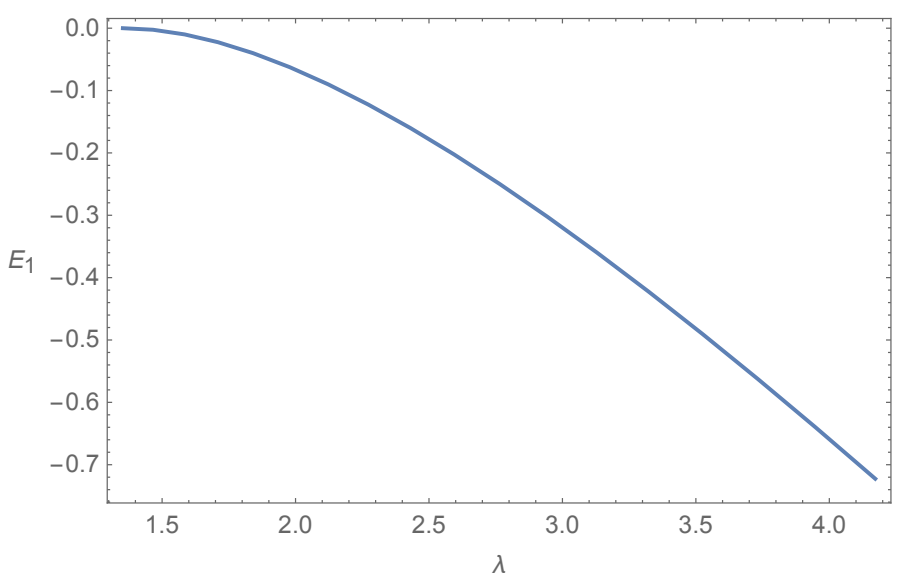

Figure 2. The energy of the first excited state $E_{1}(\lambda)=-\epsilon_{1}(\lambda)^{2}$, as a function of the coupling parameter $\lambda$.

By taking only the terms up to $\lambda$ in the expansion of the inverse, we get the equation:

$$
\frac{g_{1}(\epsilon)}{2 \pi} \lambda^{2}+\frac{f_{1}(\epsilon)}{\sqrt{2 \pi}} \lambda-1=0
$$

with

$$
\begin{gathered}
f_{1}(\epsilon)=\int_{-\infty}^{+\infty} \frac{p^{2} e^{-p^{2}}}{p^{2}+\epsilon^{2}} \mathrm{~d} p=\sqrt{\pi}-\pi \epsilon e^{\epsilon^{2}} \operatorname{erfc}(\epsilon), \\
g_{1}(\epsilon)=\int_{-\infty}^{+\infty} \mathrm{d} p \frac{p e^{-p^{2}}}{p^{2}+\epsilon^{2}} \int_{-\infty}^{+\infty} \mathrm{d} q(\sinh (p q)-p q) \frac{q e^{-q^{2}}}{q^{2}+\epsilon^{2}} .
\end{gathered}
$$

By using now the standard Taylor expansion of the hyperbolic sine, the latter double integral can be recast as the following convergent series whose coefficients are expressed in terms of the Gamma and the incomplete Gamma function:

$$
g_{1}(\epsilon)=\sum_{n=1}^{\infty} \frac{1}{(2 n+1) !}\left(\int_{-\infty}^{\infty} \frac{p^{2 n+2} e^{-p^{2}}}{p^{2}+\epsilon^{2}} \mathrm{~d} p\right)^{2}=e^{2 \epsilon^{2}} \sum_{n=1}^{\infty} \epsilon^{2(2 n+1)} \frac{\left(\Gamma(n+3 / 2) \Gamma\left(-n-1 / 2, \epsilon^{2}\right)\right)^{2}}{\Gamma(2 n+2)} .
$$

Hence, the positive solution of $(32)$ is given by

$$
\lambda_{1}(\epsilon)=\frac{2 \sqrt{2 \pi}}{\left(f_{1}^{2}(\epsilon)+4 g_{1}(\epsilon)\right)^{1 / 2}+f_{1}(\epsilon)},
$$

with domain $[0,+\infty)$ and codomain given by $\left[\lambda_{1}(0),+\infty\right), \lambda_{1}(0)$ being

$$
\lambda_{1}(0)=\frac{2 \sqrt{6}}{\sqrt{4 \pi-9}+\sqrt{3}} \approx 1.35311 .
$$

Hence, the latter function can be inverted to get $\epsilon_{1}(\lambda)$, as well as $E_{1}(\lambda)=-\epsilon_{1}(\lambda)^{2}$ whose plot is given in Figure 2

The plot of both eigenvalues $E_{0}$ and $E_{1}$ as functions of the coupling parameter $\lambda$ is shown in Figure 3 . The isolated points are those resulting from Table 1 in the aforementioned paper by Fernández [1].

\section{Conclusions}

By combining a variation of the renowned Birman-Schwinger principle and the use of Fredholm determinants, given that all the integral operators involved are positive trace class operators, we have been able to determine the two lowest eigenenergies, the one of the ground state and that of the lowest antisymmetric bound state, of the one-dimensional Hamiltonian $-\partial_{x}^{2}-\lambda e^{-x^{2} / 2}$ as functions of the coupling parameter. Whilst the ground state energy emerges out of the absolutely continuous spectrum at $\lambda=\lambda_{0}(0)=0$, the latter emerges at $\lambda=\lambda_{1}(0)$, approximately equal to 1.35311 .

The method can be further exploited to determine other eigenvalues of the Hamiltonian. For example, by starting with the function

$$
\widehat{\psi}_{2}(p)=\left(p^{2}-\frac{\epsilon(1-\epsilon)}{\sqrt{\pi} e^{\epsilon^{2}} \operatorname{erfc}(\epsilon)}\right) \frac{e^{-p^{2} / 2}}{\left(p^{2}+\epsilon^{2}\right)^{1 / 2}},
$$




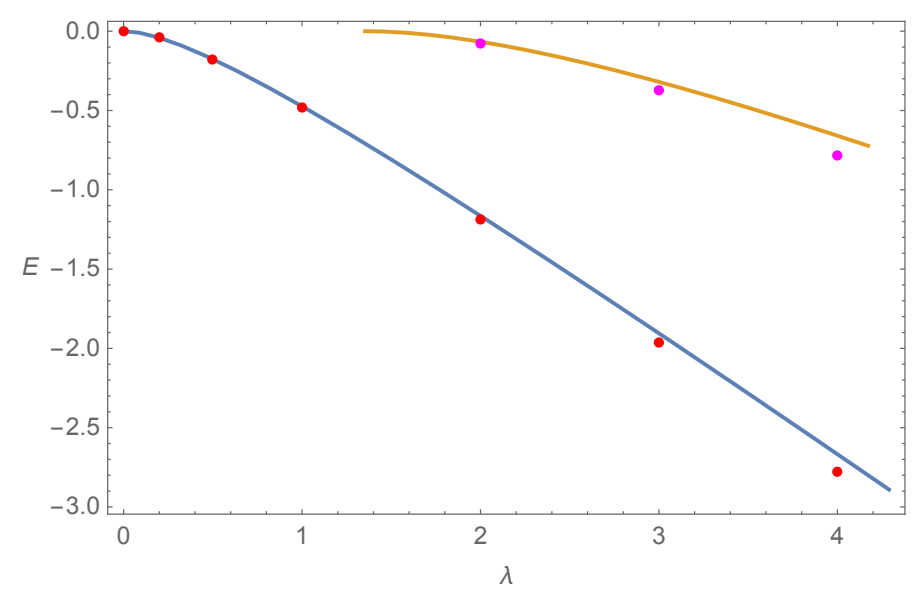

Figure 3 . The curves of both energies, $E_{0}$ (blue) and $E_{1}$ (yellow), as functions of the coupling parameter $\lambda$, and the points resulting from the table provided by Fernández in $[1$.

orthogonal to the ground state eigenfunction $\widehat{\psi}_{0}(p)=e^{-p^{2} / 2} /\left(p^{2}+\epsilon^{2}\right)^{1 / 2}$ (by using the Gram-Schmidt procedure), and the Fredholm determinant $\operatorname{det}\left(1-\lambda B_{\epsilon}^{s, 1}\right)$, one can determine the energy of the first excited symmetric bound state as a function of the coupling parameter emerging out of the absolutely continuous spectrum at the next coupling constant threshold. Work in this direction is in progress.

\section{ACKnowledgements}

Fabio Rinaldi would like to thank Prof. M. Znojil for kindly inviting him to present the main points of this article in the final session of the Conference "Analytic and Algebraic Methods in Physics" held in Prague, 11-14 September 2017. Partial financial support is acknowledged to the Spanish Junta de Castilla y León and FEDER (Project VA057U16), and MINECO (Project MTM2014-57129-C2-1-P). S. Fassari also wishes to thank the entire staff at Departamento de Física Teórica, Atómica y Óptica, Universidad de Valladolid, for their warm hospitality throughout his stay.

\section{REFERENCES}

[1] Fernández F.M.: Quantum Gaussian Wells and Barriers, Am. J. Physics, 79 (7), 2011, 752-754.

[2] Muchatibaya G., Fassari S., Rinaldi F., Mushanyu J.: A Note on the Discrete Spectrum of Gaussian Wells (I): The Ground State Energy in One Dimension, Advances in Mathematical Physics, 2016.

[3] Nandi S.: The quantum Gaussian Well, Am. J. Physics, 78, 2010, 1341-1345.

[4] Klaus M.: Some applications of the Birman-Schwinger principle, Helv. Physics Acta 55, 1982, 413-419.

[5] Klaus M.: On the bound state of Schrödinger operators in one dimension, Annals of Physics 108, 1977, $288-300$.

[6] Klaus M.: A remark about weakly coupled one-dimensional Schrödinger operators Helv. Phys. Acta 52, $1979,223$.

[7] Fassari S.: An estimate regarding one-dimensional point interactions Helv. Phys. Acta 68, 1995, 121-125.

[8] Fassari S., Rinaldi F.: On the spectrum of the Schrödinger Hamiltonian with a particular configuration of three point interactions, Reports on Mathematical Physics, 64 (3) 2009, 367-393.

[9] Reed M, Simon B.: Methods in Modern Mathematical Physics III - Scattering Theory, Academic Press, NY 1979

[10] Simon B.: The bound state of weakly coupled Schrödinger operators in one and two dimensions, Annals of Physics, 97, 1976, 279-288.

[11] Simon B.: Trace Ideals and Their Applications, Cambridge University Press, Cambridge 1979

[12] Reed M, Simon B.: Methods in Modern Mathematical Physics IV - Analysis of Operators, Academic Press, NY 1978 